
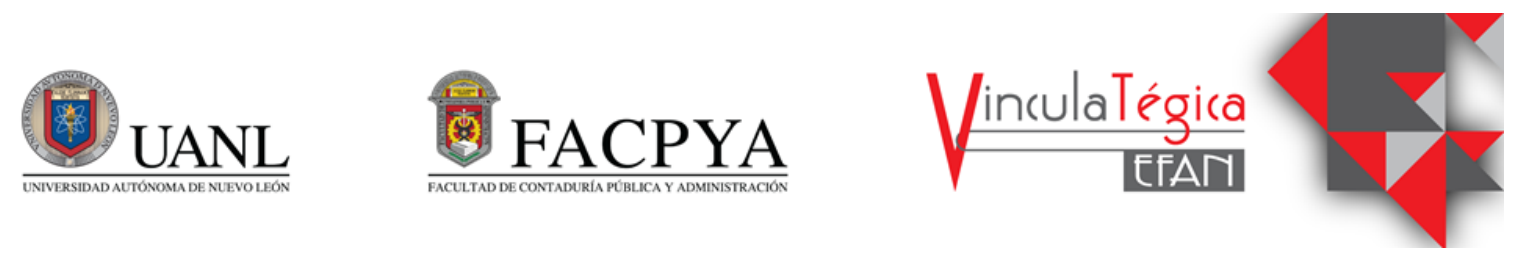

\title{
Marco teórico e instrumento de medición de las habilidades gerenciales y clima organizacional en Instituciones Bancarias de México
}

\author{
Roberto Martín Alegría Zebadúa ${ }^{1}$ y Gustavo Alarcón Martínez ${ }^{2}$ \\ ${ }^{1}$ Universidad Autónoma de Nuevo León, Facultad de Contaduría Pública y Administración. Monterrey, Nuevo \\ León, México,raz_alegria@hotmail.com, Av. Universidad S/N Col. Ciudad Universitaria, 8119991064. \\ ${ }^{2}$ Universidad Autónoma de Nuevo León, Facultad de Contaduría Pública y Administración. Monterrey, Nuevo \\ León, México, gusalamar@hotmail.com, Av. Universidad S/N Col. Ciudad Universitaria, 8180789234.
}

Información del artículo revisado por pares

Fecha de aceptación: junio-2021

Fecha de publicación en línea: diciembre-2021

DOI: https://doi.org/10.29105/vtga7.1-82

\section{Resumen}

El propósito de la presente investigación es integrar el marco teórico y el instrumento de medición que forman parte de la investigación que analiza la importancia de las habilidades gerenciales en relación al clima organizacional en las Instituciones Bancarias de México. Las habilidades gerenciales bajo estudio son la motivación, la solución de problemas, el trabajo en equipo, la comunicación efectiva y el manejo de conflictos laborales. Trabajos de investigación identificados en el marco teórico confirman la relación positiva de estas variables con el clima organizacional. La operacionalización de las variables tomo en cuenta dimensiones de definiciones teóricas, instrumentos previamente validados y la generación de ítems propios. El instrumento de medición cuenta con 46 ítems. El proceso de validez de contenido se obtuvo a través de un grupo de expertos arrojando resultados positivos. Para el nivel de confianza se utilizó el Alfa de Cronbach obteniendo niveles superiores a 0.820 para cada uno de los constructos bajo estudio.

Palabras clave: Clima organizacional, habilidades gerenciales, motivación

\begin{abstract}
The purpose of this research is to integrate the theoretical framework and the measurement instrument that are part of the research that analyzes the importance of managerial skills in relation to the organizational climate in Mexican Banking Institutions. The managerial skills under study are motivation, problem solving, teamwork, effective communication, and management of labor disputes. Research works identified in the theoretical framework confirm a positive relationship of these variables with the organizational climate. The operationalization of the variables took into account dimensions of theoretical definitions, previously validated according to the instrument and the generation of additional own items. The measuring instrument has 46 items. The content validity process was obtained through a group of experts, yielding positive results. For the confidence level, Cronbach's Alpha was estimated, obtaining levels higher than 0.820 for each one of the constructs under study.
\end{abstract}

Keywords: Organizational climate, management skills, motivation

JEL: J24 


\section{INTRODUCCIÓN}

El clima organizacional es observado y analizado por las empresas con el fin de mantenerlo en niveles positivos y de esa forma estimular la productividad de los empleados. Las empresas buscan los elementos que pudieran incidir positivamente en el clima organizacional con el fin de aportar las condiciones necesarias para lograr mejorarlo.

De acuerdo a estudios empíricos, el clima organizacional se relaciona positivamente con el nivel de productividad de los empleados. A mejor clima organizacional, mejores resultados. Derivado de lo anterior, si una empresa muestra un clima organizacional negativo, su productividad se verá disminuida y por consecuencia sus resultados y rentabilidad.

Estudios empíricos refieren a las habilidades gerenciales, el nivel de satisfacción del empleado y el estilo de liderazgo, como factores que inciden positivamente en el clima organizacional. García Solarte (2011) en su publicación, clima organizacional y su diagnóstico, cita varios modelos de diagnóstico para el clima organizacional que consideran como factores de medición a las relaciones interpersonales, condiciones físicas, normatividad interna y esquema de compensación - salarios.

Esta investigación se enfoca en la relación que guardan relaciones interpersonales, en particular las habilidades gerenciales con respecto al clima organizacional y tiene como objetivo integrar el marco teórico y desarrollar, sobre esta base, el instrumento de medición que analiza la importancia de las habilidades gerenciales en relación al clima organizacional en las Instituciones Bancarias de México. Esta investigación forma parte de una más amplia de carácter cuantitativo que se desarrollará posteriormente.

\section{MARCO TEÓRICO}

\subsection{Variable dependiente.- Clima organizacional}

El clima organizacional entendido como la percepción que los empleados tienen de los elementos que conforman el ambiente laboral de la empresa en la que trabajan y que influye en su comportamiento, se ha venido estudiado e investigando desde principios del siglo pasado. A continuación se presenta una lista de autores al respecto:

Tabla 1. Antecedentes sobre clima organizacional

\begin{tabular}{clc}
\hline 1927 & $\begin{array}{l}\text { Elton Mayo, caso Hawthorne, da inicio a la Escuela } \\
\text { Humanística de la Administración } \\
\text { Finales 30s }\end{array}$ & $\begin{array}{l}\text { Inicio del concepto del clima laboral. Se da con la teoría de } \\
\text { campo de Kurt Lewin } \\
1947\end{array}$ \\
1967 & Teoría del comportamiento o teoría behaviorista & $\begin{array}{c}\text { Santana, \& Saavedra } \\
\text { Moyano, 2015) }\end{array}$ \\
1968 & Teoría del clima organizacional de Likert & (Manzo Pinto, 2002)
\end{tabular}


Son varias las definiciones encontradas en la literatura en cuanto a clima organizacional. Una vez revisadas las definiciones contempladas por Rojas Nieto (2017), Del Toro Granados, Salazar Sotter, \& Gómez Rangel (2011), Rodríguez M., Paz Retamal, Lizana, \& Cornejo (2011), Juarez Adauta (2012), Cuadra Peralta \& Veloso Besio (2007), Arias gallegos \& Arias Cáceres (2014) y García Solarte (2011) en sus trabajos de investigación y publicaciones, se seleccionó la que contempla estas tres dimensiones: características del ambiente laboral, percepciones de los empleados sobre este ambiente y comportamiento de los empleados.

Para la presente investigación y de acuerdo a Litwin y Stringer (1968) se entenderá por clima organizacional al "conjunto de propiedades medibles del medio ambiente de trabajo, percibidas directa o indirectamente por las personas que trabajan en la organización y que influyen su motivación y comportamiento" (Rojas Nieto, 2017).

Por otra parte, estudios empíricos señalan que existe una relación positiva entre el clima organizacional y la productividad de los empleados. Korompot (2020), Managheb, Razmjooei, Gharbi, Hosseini, \& Amirianzadeh (2018), Vasudevan \& Iqbal (2018), Rodríguez M., Paz Retamal, Lizana, \& Cornejo (2011) y Del Toro Granados, Salazar Sotter, \& Gómez Rangel (2011) lo señalan en sus trabajos de investigación.

A su vez, estudios empíricos reportan relación positiva de algunas habilidades gerenciales con el clima organizacional. Nahou Larrea (2019), Rojas Nieto (2017) y Aburto Pineda (2011) relacionan positivamente la motivación con el clima organizacional. Nahou Larrea (2019), Rojas Nieto (2017) y Aburto Pineda (2011) reportan relación positiva de comunicación efectiva con el clima organizacional. Nahou Larrea (2019), Rojas Nieto (2017) y Aburto Pineda (2011) lo reportan para el manejo de conflictos laborales. Rojas Nieto (2017) para solución de problemas. Rojas Nieto (2017) y Aburto Pineda (2011) para el trabajo en equipo.

Esta investigación está dirigida a analizar la relación que guardan habilidades gerenciales claves con el clima organizacional.

\subsection{Variables independientes.- Habilidades gerenciales}

Modelos teóricos consideran a la motivación, comunicación efectiva, manejo de conflictos laborales, solución de problemas y trabajo en equipo como unas de las habilidades gerenciales clave sujetas de estudio.

Los modelos teóricos de Madrigal (2009) y Whetten y Cameron (2011) las consideran dentro de sus mediciones y evaluaciones. Estudios empíricos han encontrado relación positiva entre éstas habilidades gerenciales y el clima organizacional.

Estas habilidades gerenciales han sido estudiadas por varios autores, quienes han presentado definiciones para cada una de ellas. En este apartado se detallará el proceso de selección de la definición de la variable que será utilizada en la presente investigación y las dimensiones que la conforman. Estás dimensiones serán la base para la generación de los ítems que conformarán el instrumento de medición.

\subsubsection{Motivación}

El fenómeno de motivación ha sido estudiado a lo largo del tiempo y se han generado en torno a él varias teorías, entre las 
cuales se pueden señalar las siguientes: Jerarquía de necesidades de Maslow (1954), teoría de las motivaciones sociales de McClelland (1951,1961), teoría bifactorial de Herzberg (1959), teoría X-Y de McGregor (1960) y teoría ERC de Alderfer (1972), (Olloqui, Lopez, Peña, Cardenas, \& Ramos, Iruegas, 2011).

Así mismo se han presentado definiciones de motivación por Whetten \& Cameron (2011), Rivera Porras, Herrnández Ladine, Forgiony Santos, Bonilla Cruz, \& Rozo Sánchez (2018), Coon \& Mitterer (2010), Marin Samanez \& Placencia Medina (2017), López Mas (2005) y Madrigal Torres (2002).

Es deseable que la definición del constructo incluya el concepto de la capacidad de estimular en alguien más esa motivación. Esta condición no se incluye dentro de ninguna de las definiciones antes mencionadas. Dado lo anterior, para el presente trabajo de investigación se considera pertinente generar una definición propia basada en la literatura y que contenga este elemento.

Para la presente investigación la motivación se define como la capacidad de estimular en alguien más su voluntad y compromiso para que genere cierta actividad en aras de la búsqueda y logro de un objetivo. dimensiones:

Esta definición contiene las siguientes

- Capacidad de estimular la voluntad

- Capacidad de generar compromiso

- Capacidad de generar cumplimiento de objetivos

\subsubsection{Comunicación efectiva}

La revisión de literatura arroja diversas definiciones de comunicación efectiva, entre ellas las consideradas por Medina (2016), Whetten y Cameron (2011) y Madrigal Torres (2002) en sus publicaciones.

Considerando lo anterior, para el presente trabajo de investigación y de acuerdo a Harold y Heinz (1991), la comunicación efectiva se puede definir como el intercambio de información, ideas o experiencias de una mente a otras. Un gerente puede transmitirlas y la efectividad marcara la realización administrativa (Medina, 2016).

La presente definición contempla las siguientes dimensiones:

- Capacidad de intercambiar información

- Capacidad de transmitir ideas y experiencia

- Nivel de realización administrativa

\subsubsection{Manejo de conflictos laborales}

Analizando las definiciones utilizadas por Whetten \& Cameron (2011) y por Madrigal Torres (2002) se aprecian las siguientes dimensiones: definición de conflicto laboral y fases para su manejo. Por lo anterior, se consideró pertinente generar una definición propia basada en lo encontrado en la literatura que contemple ambos elementos.

Para este trabajo de investigación el manejo de conflictos laborales se define como la capacidad gerencial de concertar acuerdos sobre diferencias presentadas en los procesos inherentes al trabajo de los empleados, cumpliendo las fases de diagnóstico del origen del conflicto, estrategia para abordarlo, ejecución de la estrategia y solución exitosa del conflicto.

Esta definición contiene las siguientes dimensiones: 
- Capacidad de diagnosticar el origen del conflicto

- Capacidad de generar una estrategia de solución

- Capacidad de ejecutar la estrategia

- Capacidad de generar acuerdos

\subsubsection{Solución de problemas}

Considerando la definición incluida por Madrigal Torres (2002), Acevedo Borrego, Linares Barrantes, \& Cachay Boza (2010) y Whetten \& Cameron (2011) en sus investigaciones, encontramos dos dimensiones: la descripción del significado de problema y los pasos a seguir para la solución de los mismos. Por lo anterior, se procedió a generar una definición propia en base a la literatura que consolidara las dos dimensiones.

Para la presente investigación la solución de problemas es la capacidad de modificar ciertas condiciones no deseadas del estado actual y obtener las condiciones deseadas o predefinidas, cumpliendo los siguientes pasos: identificación y definición del problema, generar soluciones alternativas, evaluar y seleccionar una alternativa, poner en práctica la solución y hacer un seguimiento.

La presente definición contempla las siguientes dimensiones:

- Capacidad de identificar y definir el problema.

- Capacidad de generar soluciones alternativas

- Capacidad de evaluar y seleccionar una alternativa
- Éxito en la solución del problema

\subsubsection{Trabajo en equipo}

Se analizaron las definiciones utilizadas por Medina Fuenmayor (2010), Whetten \& Cameron (2011) y Madrigal Torres (2002) en sus publicaciones.

Considerando lo anterior, para la presente investigación, trabajo en equipo se define como el conjunto de personas que trabajan juntas para obtener las mismas metas. Se privilegia las metas comunes sobre las individuales. Contempla que la suma de los conocimientos, experiencias y habilidades de los integrantes del grupo potencializa el resultado. Se asociaría al término de sinergia (Medina Fuenmayor, 2010).

La definición seleccionada contiene las siguientes dimensiones:

- Fomentar que las personas trabajen juntas buscando metas comunes.

- Privilegia las metas comunes sobre las individuales.

- Pondera la aportación de conocimiento, experiencia $\mathrm{y}$ habilidades propias al grupo

\subsection{Hipótesis}

La motivación, la comunicación efectiva, el manejo de conflictos laborales, la solución de problemas y el trabajo en equipo son habilidades gerenciales que inciden positivamente al clima organizacional

Figura 1. Modelo gráfico de hipótesis

Habilidades gerenciales

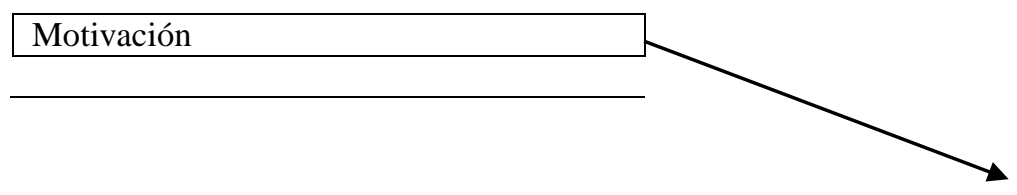




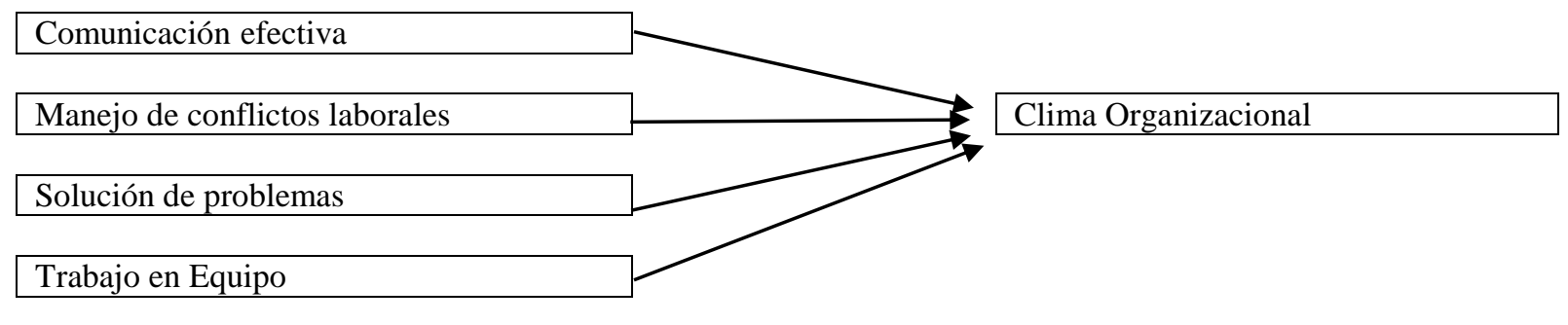

Fuente: Elaboración propia

\section{Metodología}

\subsection{Operacionalización inicial de las variables}

La operacionalización de las variables conlleva medir un concepto y posteriormente aplicar esa medición dentro del proceso de investigación (Mendoza \& Garza, 2009). El proceso de operacionalización se fundamenta tanto en la definición conceptual de la variable como en la operacional. Es decir, se transita de la definición de la variable a través de sus componentes, dimensiones e indicadores, hasta los ítems con los cuales podrán ser medidas (Hernández Sampieri, Fernández Collado, \& Baptista Lucio, 2014).

El proceso de operacionalización inicial implicó lo siguiente:

1. Se analizaron definiciones de cada una de las variables implicadas en el presente estudio y se definió cual sería utilizada para cada una de ellas.

2. Adicionalmente se analizaron estudios aplicados que confirman la vinculación entre las variables independientes y la dependiente.

3. El análisis de los puntos anteriores permitió definir las dimensiones para el análisis específico de las variables. Se tomaron en cuenta las dimensiones que conforman las definiciones y las describen de mejor manera.
4. Se identificaron en la literatura instrumentos desarrollados para medir la variable dependiente y las independientes consideradas en la presente investigación.

5. Se tomaron los ítems de los instrumentos identificados en la literatura y como complemento, se procedió a elaborar ítems propios para las variables independientes en función de las dimensiones identificadas de acuerdo con las definiciones previamente seleccionadas para cada una de las variables.

6. Para las variables independientes se compilaron el total de ítems identificados en la literatura, así como los propios en un solo listado, procediendo a seleccionar para cada una de las variables los ítems que representan de mejor manera las dimensiones de los constructos objeto de estudio.

7. Para la variable dependiente se seleccionó el instrumento de Litwin y Stringer (1968). El instrumento contaba con nueve dimensiones las cuales fueron reducidas a cinco, seleccionado las de mayor pertinencia para la presente investigación. Así mismo, los ítems se redujeron a doce.

8. La redacción de los ítems fue ajustada y modificada de acuerdo con la orientación de la presente investigación, habilidades gerenciales, y al sujeto de 
estudio que en este caso son los responsables de las áreas de recursos humanos, operaciones y comercial de los bancos.

Sobre esta base se llevó a cabo la primera etapa de la operacionalización de las variables. La según etapa contempló poner a consideración de los expertos la validación de la encuesta.

\subsection{Validación de expertos}

Para confirmar la validez de contenido se acudió a un grupo de expertos, el cual evaluó y calificó que tanto mide un instrumento la variable que se pretende analizar (Hernández Sampieri, Fernández Collado, \& Baptista Lucio, 2014). El proceso de validez pretende reducir el error no aleatorio, es decir, que los indicadores contenidos en el instrumento no representen a un concepto diferente del concepto teórico que se pretende medir (Mendoza \& Garza, 2009).

Como ya se mencionó la validez de contenido del instrumento se realizó a través un grupo de expertos conformado de la siguiente manera:

Dr. Joel Mendoza Gómez, PHD en Administración, Profesor investigador para la Universidad Autónoma de Nuevo León, área de estudio Capital Humano.

Dr. Pablo Guerra Rodríguez, PHD en Administración, PHD en Administración del talento humano, docente e investigador para la Universidad Autónoma de Nuevo León, Líder de cuerpo académico 365 Estrategias en Mercadotecnia y Modelos de negocios.

Dr. José Luis Montes Martínez, PHD en Economía y dirección de empresas, Maestría en Desarrollo Organizacional, Licenciado en Psicología, Director de la carrera de Licenciado en Psicología Organizacional, Tecnológico de Monterrey, Campus Monterrey.

Lic. Cesar Hernández Cabrera, Maestría en desarrollo de capital humano, Maestría en antropología y ética, Director de Programas, Profesor y Jefe de Academia de recursos humanos para ICAMI (Centro de Formación y Perfeccionamiento Directivo), Consultor Jurídico Laboral y de Desarrollo Humano.

El proceso de validación de contenido implicó clasificar cada uno de los ítems de acuerdo a su relevancia en una de las siguientes categorías: 1 Irrelevante, 2 Poco relevante, 3 Relevante, 4 Muy relevante.

El resultado arroja que el promedio de las ponderaciones medias de los ítems de cada variable evaluada por los expertos tuvieron puntuaciones que van de 3.2 a 3.5, con una puntuación promedio máxima por ítem de 3.8 y una mínima de 2.5 , ver tabla 2. De acuerdo con este resultado no se eliminó ninguno de los ítems, de tal manera que se consideró la encuesta validada.

Tabla 2. Validez de contenido:

\begin{tabular}{lcccc}
\hline \multicolumn{1}{c}{ Variable } & No de ítems & Promedio & Máxima & Mínima \\
\hline Clima organizacional & 12 & 3.4 & 3.8 & 2.5 \\
Motivación & 7 & 3.6 & 4.0 & 3.3 \\
Solución de problemas & 7 & 3.4 & 3.8 & 2.8 \\
Trabajo en equipo & 7 & 3.5 & 3.8 & 3.0 \\
Comunicación efectiva & 7 & 3.5 & 4.0 & 3.3
\end{tabular}


Manejo de conflictos laborales

6

3.5

4.0

3.0

Fuente: elaboración propia

\section{Resultados}

\subsection{Operacionalización versión final}

La operacionalización arrojó un cuestionario que incluye 12 ítems para la variable dependiente clima organizacional y 34 ítems para las variables independientes motivación, comunicación efectiva, manejo de conflictos laborales, solución de problemas y trabajo en equipo.
Del conjunto de ítems, $89 \%$ provinieron de instrumentos utilizados y validados en investigaciones empíricas identificadas en la literatura; $11 \%$ fue conformado por ítems propios.

La operacionalización final se presenta en las tablas 3 al 8 en las que se destacan las definiciones de cada variable, las dimensiones y los ítems considerados, así como las fuentes documentales correspondientes.

Tabla 3. Operacionalización de variable dependiente clima organizacional

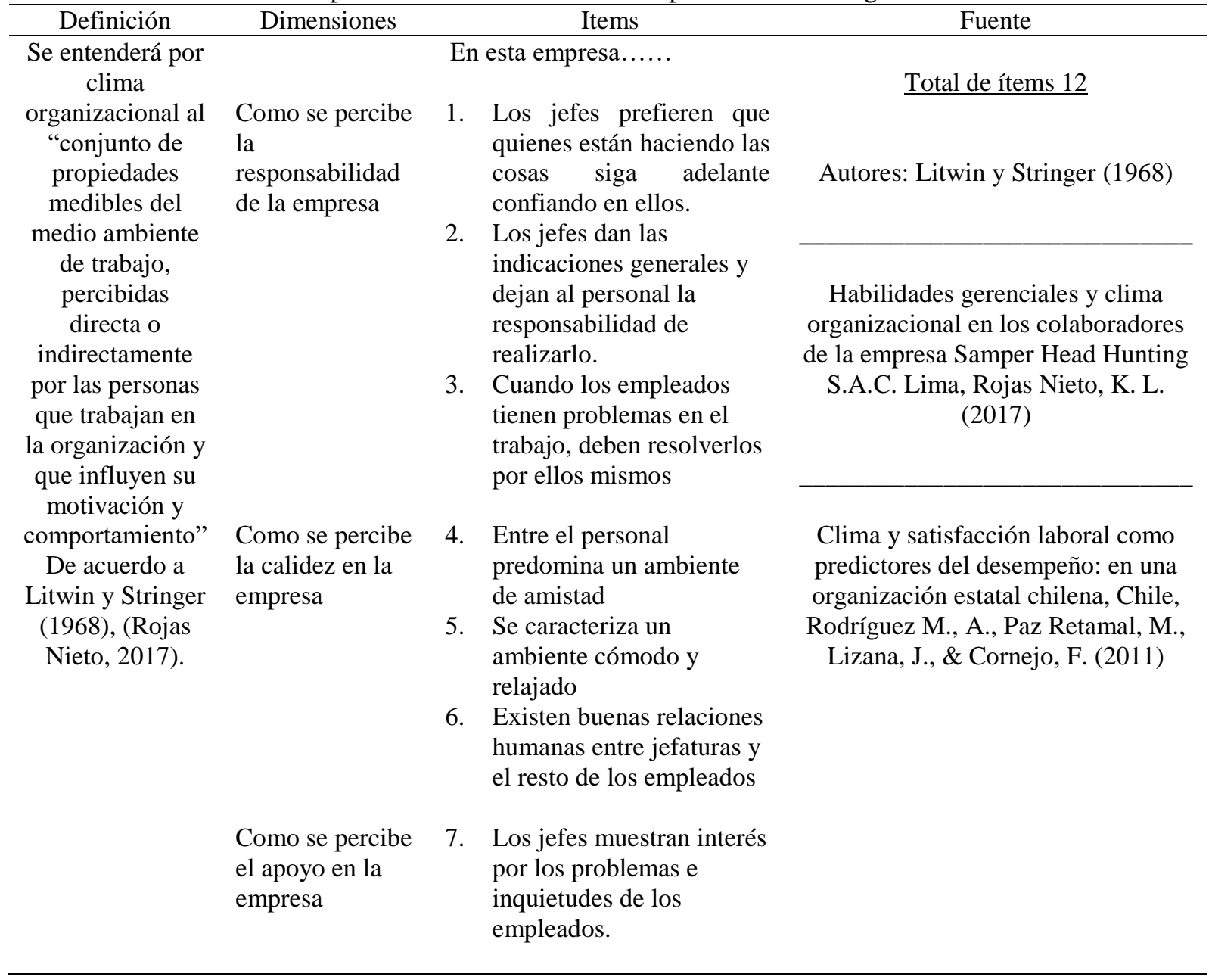


Como se percibe el conflicto en la empresa

Como se percibe la identidad en la empresa
8. Cuando el empleado tiene que hacer un trabajo difícil, puede contar con la ayuda de los jefes.

9. Los jefes aprovechan las discrepancias entre los empleados para crear oportunidades.

10. Se alienta a los empleados para que digan lo que piensan, aunque no coincidan con su jefe.

11. Los empleados están satisfechos de estar en esta organización

12. Los empleados sienten que pertenecen a un grupo de trabajo que funciona satisfactoriamente.

Tabla 4. Operacionalización de variable independiente motivación

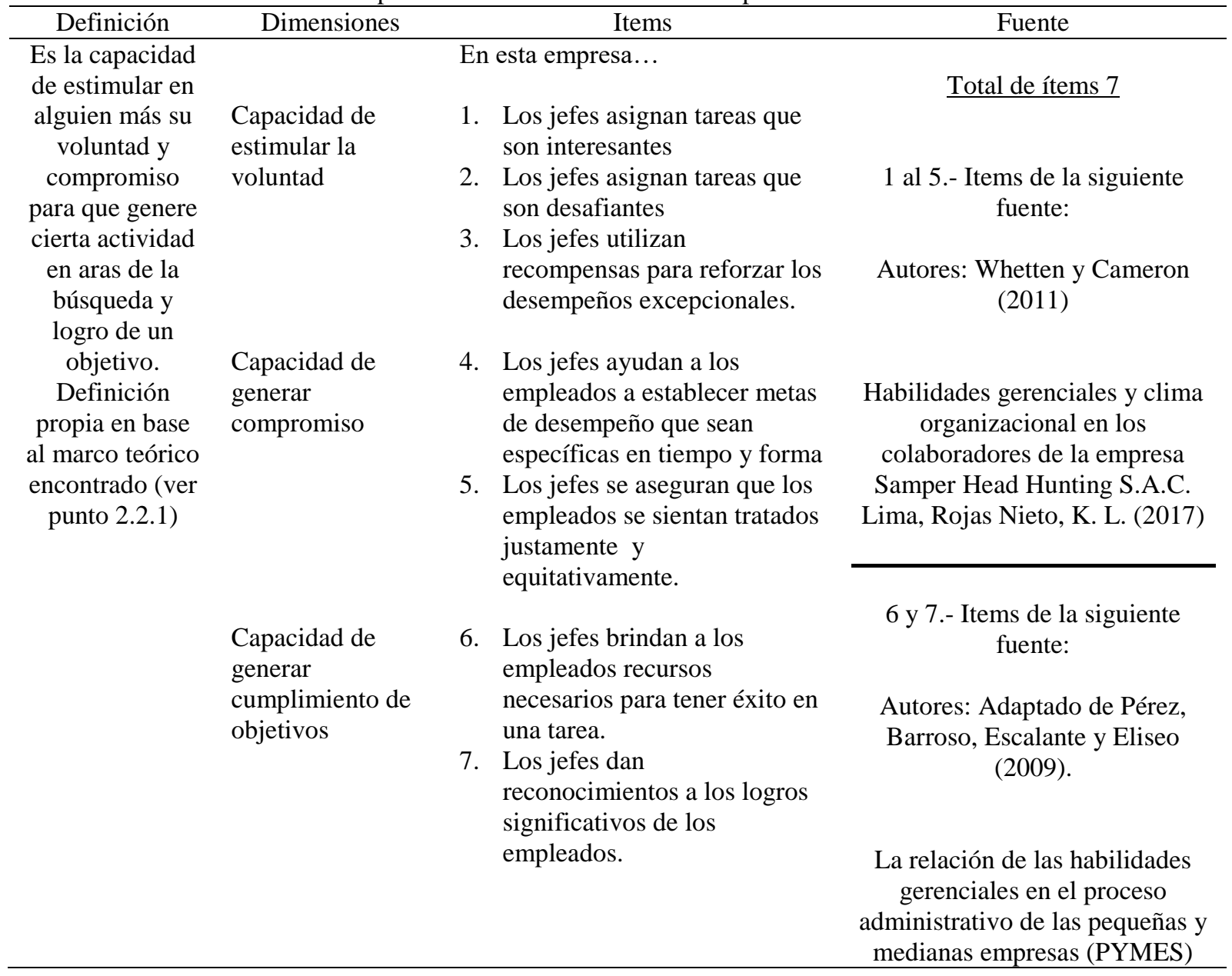


dedicadas al comercio ubicadas

en ciudad Obregón, Sonora,

México, Paredes Zempual, D.

(2019).

Fuente: elaboración propia

Tabla 5. Operacionalización de variable independiente solución de problemas

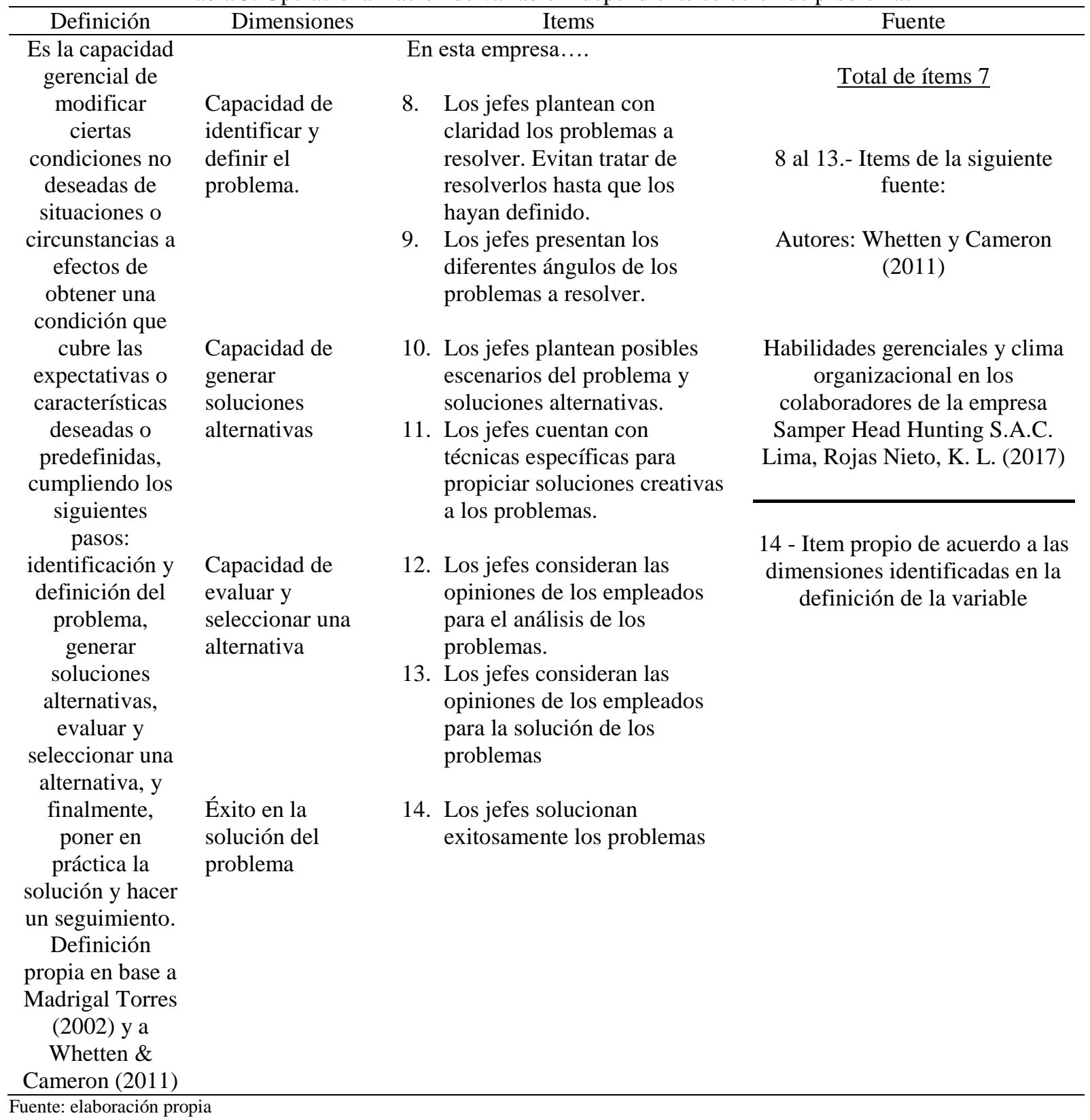

Tabla 6. Operacionalización de variable independiente trabajo en equipo

Definición Dimensiones $\quad$ Items




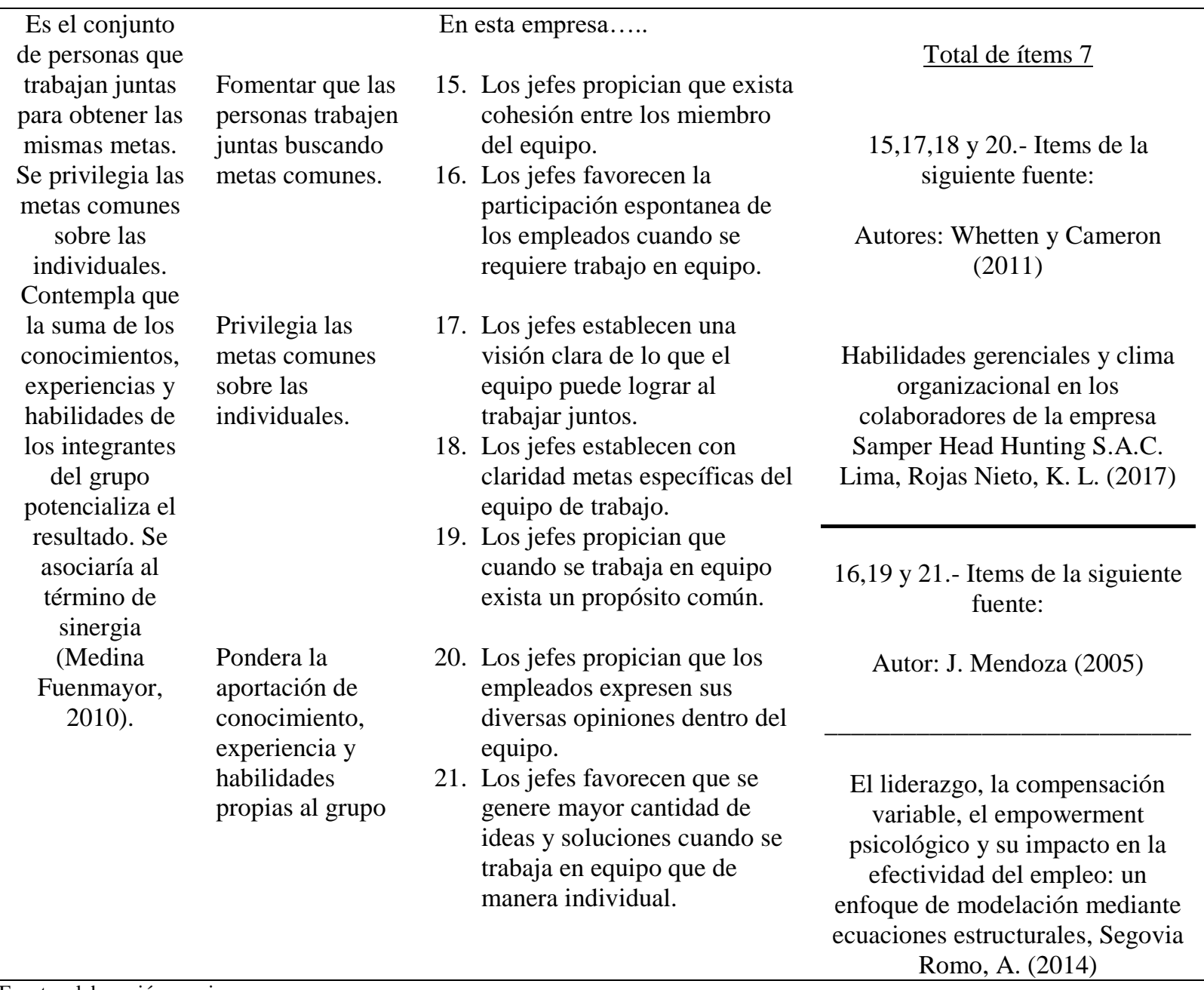

Fuente: elaboración propia

Tabla 7. Operacionalización de variable independiente comunicación efectiva

\begin{tabular}{|c|c|c|c|}
\hline Definición & Dimensiones & Items & Fuente \\
\hline \multirow{8}{*}{$\begin{array}{c}\text { Según Harold y } \\
\text { Heinz (1991) se } \\
\text { puede definir } \\
\text { como el } \\
\text { intercambio de } \\
\text { información, } \\
\text { ideas o } \\
\text { experiencias de } \\
\text { una mente a } \\
\text { otras. Un } \\
\text { gerente puede } \\
\text { transmitirlas y } \\
\text { la efectividad } \\
\text { marcara la } \\
\text { realización } \\
\text { administrativa } \\
\text { (Medina, 2016). }\end{array}$} & & En esta empresa.... & \\
\hline & & & Total de ítems 7 \\
\hline & $\begin{array}{l}\text { Capacidad de } \\
\text { intercambiar }\end{array}$ & $\begin{array}{l}\text { 22. Los jefes son receptivos para } \\
\text { intercambiar puntos de vista }\end{array}$ & \\
\hline & información & con los empleados & $23,24,25,27$ y $28 .-$ Items de la \\
\hline & & $\begin{array}{l}\text { 23. Los jefes son claros al } \\
\text { orientar a los empleados sobre }\end{array}$ & ciouichte funta. \\
\hline & & las áreas & Autores: Whetten y Cameron \\
\hline & & $\begin{array}{l}\text { 24. Los jefes transmiten con } \\
\text { claridad las instrucciones a } \\
\text { los empleados para que } \\
\text { realicen sus tareas. }\end{array}$ & (2011) \\
\hline & $\begin{array}{l}\text { Capacidad de } \\
\text { transmitir ideas y } \\
\text { experiencia }\end{array}$ & $\begin{array}{l}\text { 25. Los jefes dan } \\
\text { retroalimentación objetiva a } \\
\text { los empleados en relación a } \\
\text { las actividades que deben } \\
\text { realizar. }\end{array}$ & $\begin{array}{l}\text { Habilidades gerenciales y clima } \\
\text { organizacional en los } \\
\text { colaboradores de la empresa } \\
\text { Samper Head Hunting S.A.C. } \\
\text { Lima, Rojas Nieto, K. L. (2017) }\end{array}$ \\
\hline
\end{tabular}


Nivel de realización administrativa
26. Los jefes transmiten con claridad instrucciones que deberán llevar a cabo los empleados.

27. Los jefes toman en cuenta las opiniones de los empleados cuando se analiza una situación.

28. Los jefes toman en cuenta las opiniones de los empleados para su toma de decisiones.
22 y 26.- Items propios de acuerdo a las dimensiones identificadas en la definición de la variable

Fuente: elaboración propia

Tabla 8. Operacionalización de variable independiente manejo de conflictos laborales

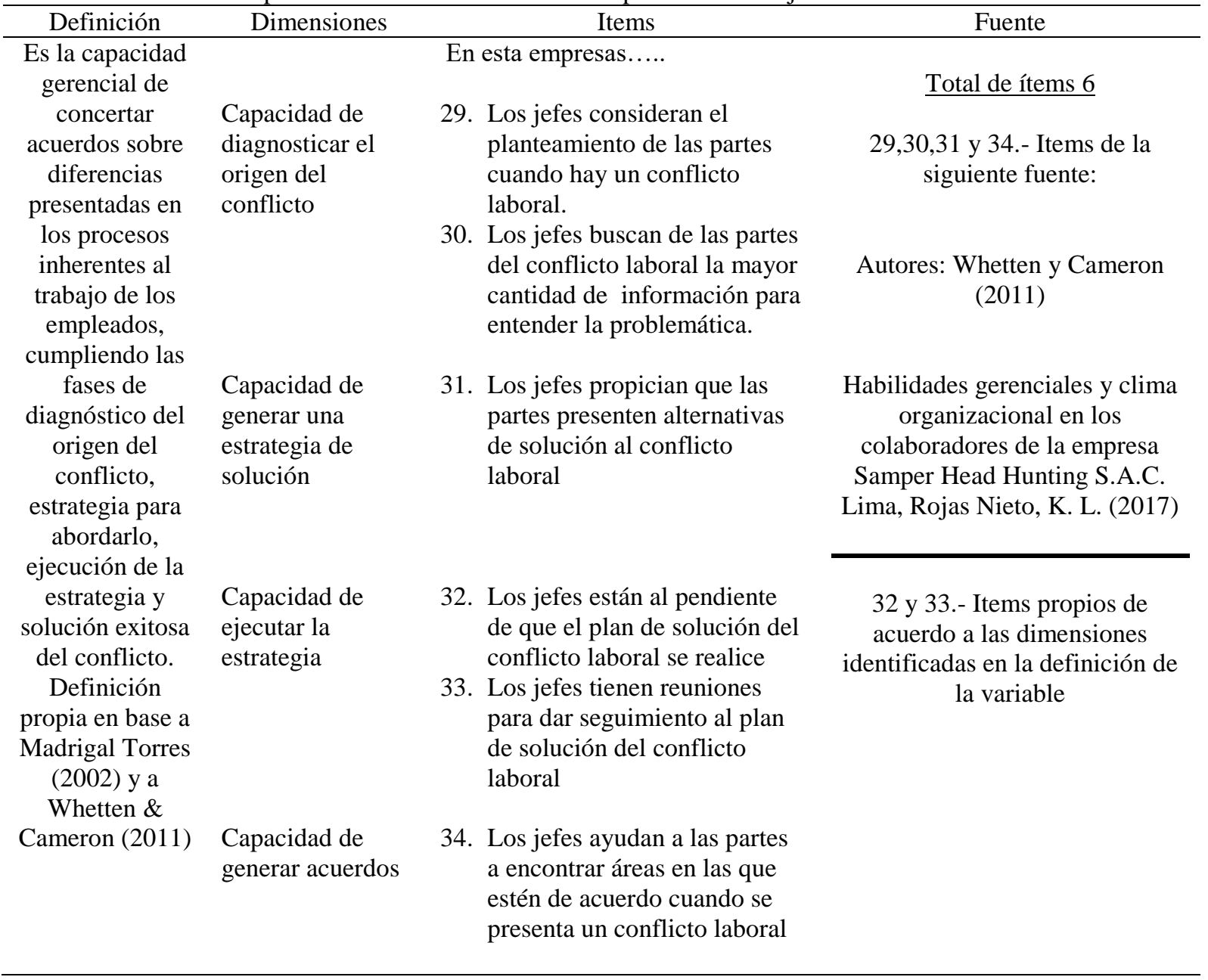

Fuente: elaboración propia

\subsection{Análisis de confiabilidad}


El paso final para el establecimiento del instrumento de medición fue el análisis de confiabilidad, que permitió confirmar el grado de fiabilidad del instrumento; es decir, hasta donde los ítems que conforman el instrumento están midiendo la misma variable. En la medida en que los ítems midan lo mismo, existirá una alta correlación entre ellos. El Alfa de Cronbach mide el grado de ésta correlación (Mendoza \& Garza, 2009).

Se realizó una prueba piloto que contempló 52 registros del instrumento de medición. Se utilizó escala tipo Likert. Para clima organizacional considera una medición de 1 para totalmente en desacuerdo, 2 en desacuerdo, 3 indiferente, 4 de acuerdo y 5 totalmente de acuerdo. Para las variables independientes van de 1 Nunca, 2 Casi nunca, 3 A veces, 4 Casi siempre y 5 Siempre. Escalas similares fueron utilizadas en estudios como el de Peña Cárdenas, Díaz Díaz, \& Olivares Medina (2015), Aburto Pineda (2011), Paredes Zempual (2019), Rojas Nieto (2017) y Segovia Romo (2014).

Para el análisis de confiabilidad se estimó el Alfa de Cronbach. Dicha estimación arrojo Alfas de Cronbach entre .828 y .937 los cuales se consideran confiables de acuerdo a los rangos utilizados por autores como Segovia Romo (2014) y Paredes Zempual (2019). Véase tabla 9.

Tabla 9. Alfa de Cronbach

\begin{tabular}{lcc}
\hline \multicolumn{1}{c}{ Variable } & \# de Ítems & Alfa de Cronbach \\
\hline Clima organizacional & 12 & 0.828 \\
Motivación & 7 & 0.845 \\
Solución de problemas & 7 & 0.870 \\
Trabajo en equipo & 7 & 0.915 \\
Comunicación efectiva & 7 & 0.899 \\
Manejo de conflictos laborales & 6 & 0.937 \\
\hline
\end{tabular}

Fuente: elaboración propia a partir de los datos recolectados

\section{Conclusiones}

El clima organizacional ha sido objeto de estudio y existen teorías, modelos y estudios empíricos relacionados a él. En ellos se confirma que el clima organizacional se relaciona positivamente con el desempeño de los empleados y su productividad.

Estudios empíricos muestran a la motivación, la comunicación efectiva, el manejo de conflictos laborales, la solución de problemas y el trabajo en equipo, como habilidades gerenciales que inciden positivamente en el clima organizacional.

El proceso de operacionalización final permitió integrar los elementos necesarios para conformar el instrumento de medición relativa a las variables que serán objeto de estudio. El instrumento de medición generado cumple con el proceso de validez de expertos y con el de confiabilidad mostrando niveles de Alfa de 
Cronbach superiores a 0.820 para cada uno de los constructos.

El marco teórico, el proceso de operacionalización y el instrumento derivados de ellos presentados en este documento, formarán parte de una investigación más amplia, que considere un análisis cuantitativo en relación al impacto de las habilidades gerenciales sobre el clima organizacional. 


\section{Referencias}

Aburto Pineda, H. I. (2011). Habilidades directivas: Determinantes en el clima organizacional . Investigación y Ciencia, vol. 19, núm. 51, 41-49.

Acevedo Borrego, A., Linares Barrantes, C., \& Cachay Boza, O. (2010). Herramienta para superar el dilema gerencial: Toma de decisiones o resolución de problemas. Industrial Data, 18-27.

Arias gallegos, W., \& Arias Cáceres, G. (2014). Relación entre el clima organizacional y la satisfacción laboral en una pequeña empresa del sector privado . Ciencia y trabajo, 185-191.

Coon, D., \& Mitterer, J. (2010). INtroducción a la Psicología. México, D.F.: Cengage Learning Editores.

Cuadra Peralta, A., \& Veloso Besio, C. (2007). Liderazgo, clima y satisfacción laboral en las organizaciones. Universum, 43-58.

Del Toro Granados, J., Salazar Sotter, M. C., \& Gómez Rangel, J. (2011). Clima organizacional, satisfacción laboral y su relación con el desempeño laboral en trabajadores de una Pyme de servicios de ingienería. Clío América, 204-227.

Ganga Contreras, F. A., Piñones Santana, M. A., \& Saavedra Moyano, L. (15 de Febrero de 2015). Espol, Facultad de Ciencias Sociales y Humanísticas. Obtenido de Espol, Facultad de Ciencias Sociales y Humanísticas Web site: http://www.fcsh.espol.edu.ec/es/clima-organizacionalalgunos-basamentos-hist\%C3\%B3ricos-y-conceptuales-para-la-reflexi\%C3\%B3n-porfrancisco

García Solarte, M. (2011). Clima Organizacional y su diagnóstico: Una aproximación conceptual. Cuadrenos de administración, 43-61.

Hernández Sampieri, R., Fernández Collado, C., \& Baptista Lucio, M. (2014). Metodología de la investigación. México D.F.: McGRAW-HILL / INTERAMERICANA EDITORES.

Juarez Adauta, S. (2012). Clima organizacional y satisfacción laboral. Revista médica del Instituto Mexicano del Seguro Social, 307-314.

Korompot, A. (2020). Influence of Motivation and Work Environment The Performance of Employees Pt. Multi Putra Makmur . American Journal of Multidisciplinary Research \& Development, 2125.

López Mas, J. (2005). Motivación laboral y gestión de recursos humanos en la teoría de Federick Herzberg. Gestión en el tercer milenio, 25-36.

Madrigal Torres, B. E. (2002). Habilidades directivas. Mexico, D.F.: Mc Graw Hill.

Managheb, S. E., Razmjooei, P., Gharbi, M. R., Hosseini, M., \& Amirianzadeh, M. (2018). Mediating role of organizational silence in the relationship between organizational climate and job performance . Amazonia Investiga, 72-86.

Manzo Pinto, J. (2002). El legado de Frederick Irving Herzberg. Revista Universidad EAFIT, 79-86.

Marin Samanez , H. S., \& Placencia Medina, M. D. (2017). Motivación y satisfacción laboral del personal de una organización de salud del sector privado . Horiz Med , 42-52.

Medina Fuenmayor, M. (2010). Talento humano y trabajo en equipo del personal directivo de las universidades del municipio Maracaibo. Telos, 79-97.

Medina, A. (2016). Plan estratégico de comunicación efectiva, para mejorar el ambiente de trabajo en la escuela estatal Efrain Pérez del municipio Libertador Estado Carabobo (Tesis de Maestría). Valencia.

Mendoza, J., \& Garza, J. (2009). La medición en el proceso de investigación científica: Evaluación. InnOvaciOnes de NegOciOs, 17-32.

Nahou Larrea, M. (2019). Propuesta de un modelo de competencias directivas como factor de influencia en el clima organizacional de una institución de educación superior en Xalapa, Veracruz. Período 2016-2020 . Ciencia Administrativa, 92-100. 
Nita, M., \& Stanciu, R. (2015). The influence of the management communication on the organizational culture and climate. Annals of the oradea university, Fascicle of Management and Technological Engineering, Issue \#1. doi:10.15660/AUOFMTE.2015-1.3132

Olloqui, Lopez, A. M., Peña, Cardenas, M. C., \& Ramos, Iruegas, E. M. (2011). Factores de satisfacción dentro del clima laboral de una Pyme. Global Conference on Business and Finance Proceedings $\downarrow$ Volume $6 \diamond$ Number 2, 828.

Paredes Zempual, D. (2019). La relación de las habilidades gerenciales en el proceso administrativo de las pequeñas y medianas empresas (PYMES) dedicadas al comercio ubicadas en ciudad Obregón, Sonora, México, 2017 (Tesis de Doctorado). San Nicolas de los Garza, N.L.

Rivera Porras, D. A., Herrnández Ladine, J. D., Forgiony Santos, J. O., Bonilla Cruz, N. J., \& Rozo Sánchez, A. C. (2018). Impacto de la motivación laboral en el clima organizacional y las relaciones interpersonales en los funcionarios del sector salud. Espacios, 17.

Rodríguez M., A., Paz Retamal, M., Lizana, J., \& Cornejo, F. (2011). Clima y satisfacción laboral como predictores del desempeño: en una organización estatal chilena. Salud \& Sociedad, 219234.

Rojas Nieto, K. L. (2017). Habilidades gerenciales y clima organizacional en los colaboradores de la empresa Samper Head Hunting S.A.C. (Tesis de Maestria). Universidad César Vallejo, Lima.

Salazar Estrada, J. G., Guerrero Pupo, J. C., Machado Rodríguez, Y. B., \& Cañedo Andalia, R. (2009). Clima y cultura organizacional: dos componentes esenciales en la productividad laboral. ACIMED, 67-75.

Segovia Romo, A. (2014). El liderazgo, la compensacion variable, el empowerment psicológico y su impacto en la efectividad del empleo: un enfoque de modelación mediante ecuaciones estructurales (Tesis Doctoral). San Nicolas de los Garza, N.L.

Vasudevan, L., \& Iqbal, A. (2018). Relationship between organizational climate and citizenship behavior of the non-supervisory staff in Bank Simpanan National, Malaysia . International Journal of Human Capital in Urban Management, 287-302.

Whetten, D. A., \& Cameron, K. S. (2011). Desarrollo de habilidades directivas. México: Pearson Educación. 